\title{
Predictors of good response to endovascular treatment of posterior circulation stroke.
}

Gramegna Laura Ludovica ${ }^{1}$, Requena Manuel ${ }^{2}$, Dinia Lavinia ${ }^{1}$, Melendez Fernando ${ }^{1}$, Hernández David ${ }^{1}$, Coscojuela Pilar ${ }^{1}$, Quintana Manuel ${ }^{3}$, Vert Carla ${ }^{4}$, Rubiera Marta², Ribo Marc², Rovira Alex4, Molina Carlos², Tomasello Alejandro1.

'Section of Interventional Neuroradiology, ${ }^{4}$ Section of Neuroradiology and Magentic Resonance Unit, Department of Radiology, Vall d'Hebron University Hospital, Barcelona, Spain.

${ }^{2}$ Stroke Unit and ${ }^{3}$ Epilepsy Unit, Department of Neurology, Vall d'Hebron University Hospital, Barcelona, Spain.

\section{Introduction}

Posterior circulation stroke represents approximately $20 \%$ of all ischemic strokes and is associated with bad outcome in $68 \%$ $80 \%$ of patients. Endovascular treatment is considered a reasonable approach in patients with acute posterior circulation strokes ${ }^{1}$, but it remains unclear which category of patients benefits the most after endovascular treatment.

\section{Material and Methods}

We evaluated consecutive patients with acute stroke in the posterior circulation who underwent endovascular treatment from January 2015 to December 2017. Good outcome was defined as a modified Rankin Scale (mRS) score 0-3 at three months, in accordance with the definition used in the BASICS registry ${ }^{2}$. Intracranial atheromatosis was recorded as previously reported ${ }^{3}$. The association between clinical and angiographical signs and good outcome was assessed with uni and multivariable analysis.

\section{Results}

We included 49 consecutive patients (mean age, $70 \pm 12$ years; median NIHSS, 16 IR8-30). In univariate analysis, age ( $p=0.01$ ), active smoking $(p=0.04)$, hypertension $(p=0.03)$, absence of intracranial atheromatosis $(p=0.03)$, successful recanalization $(p=0.04)$ and number of passes needed to achieve recanalization $(p=0.01)$ were significantly associated with good outcomes. In multivariate analysis, the absence of intracranial atheromatosis (odd ratio $=6.450,95 \% \mathrm{Cl}: 1.088-38.240, \mathrm{p}=0.040$ ) and age $<70$ (odd ratio $=6.2,95 \% \mathrm{Cl}: 1.516-25.465, \mathrm{p}=0.01$ ) were independently associated with good outcome at three months .

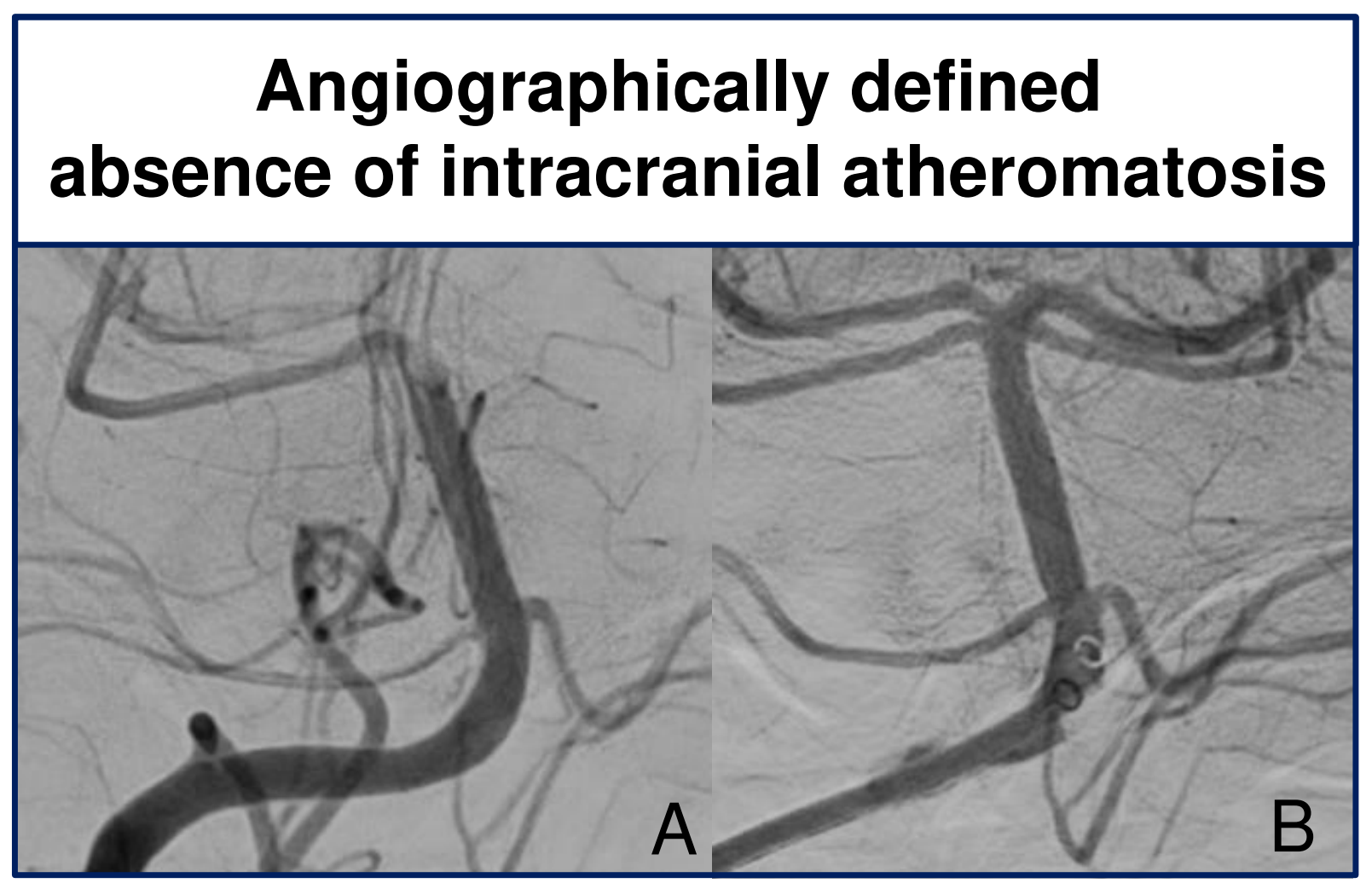

Figure 1: Antero-posterior digital subtraction angiography of a 70 years female patient. A: Diagnostic angiography showing a filling defect at the top of basilar, with no other appreciable vascular irregularity. B: Final angiography with no evidence of focal stenosis after recanalization. mRS at three months: 1 .

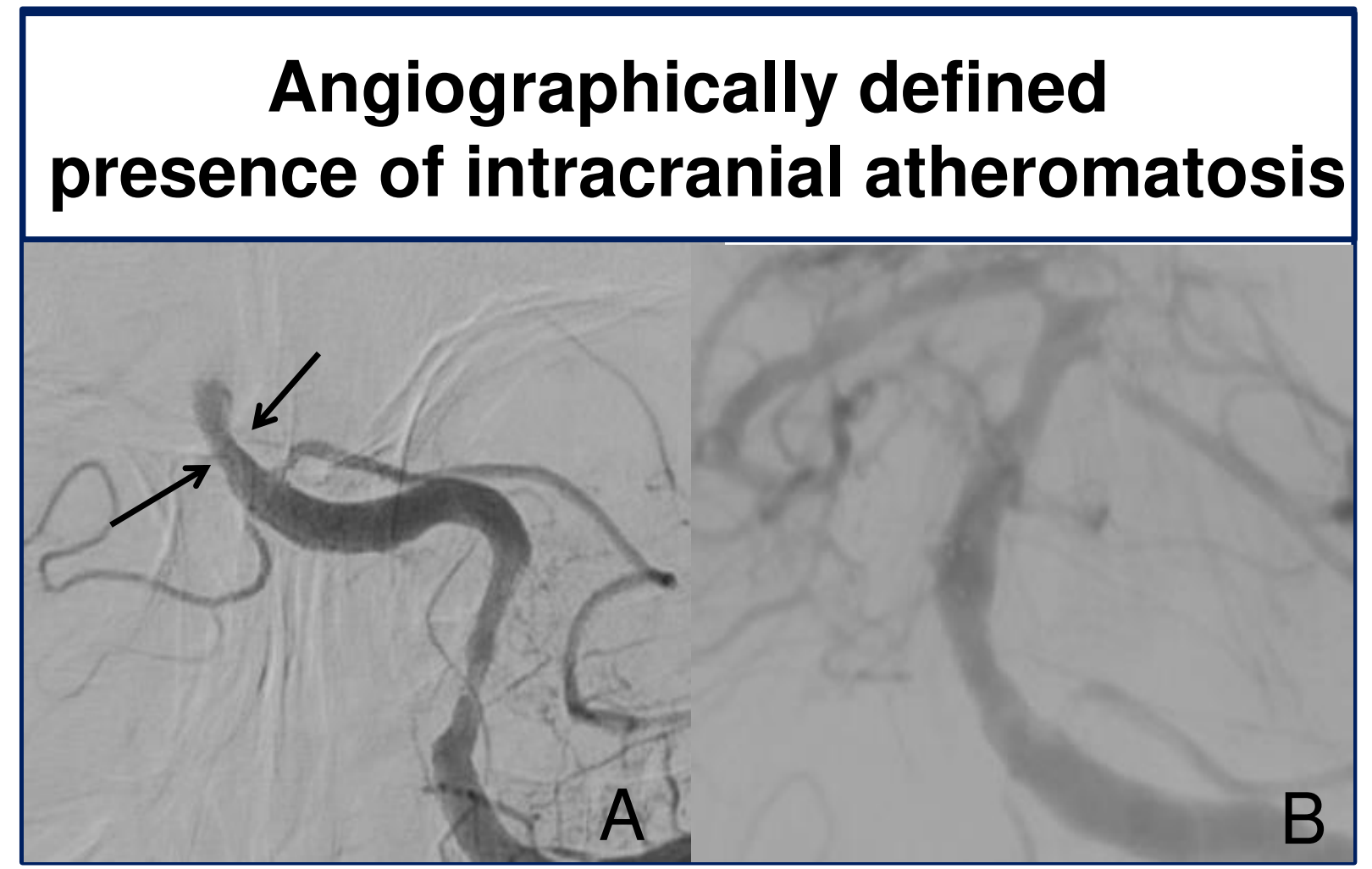

Figure 2: Antero-posterior digital subtraction angiography of a 83 years female patient. A: Diagnostic angiography showing a focal luminal narrowing highly indicative of intracranial atheromatosis. B: Final angiography with diffuse luminal irregularities after recanalization. mRS at three months: 6 .

\section{Conclusion}

In this study, patients with angiographically-defined absence of intracranial atherosclerosis were good responders to endovascular treatment, whereas patients with sign of intracranial atheromatosis were not. Clinical trials to determine whether new treatment protocols may be more efficient in patients with intracranial atheromatosis are urgently needed.

\section{Bibliography}

